Urologe 2009 $488: 709$

DOI 10.1007/s00120-009-2009-1

Online publiziert: 17. Juni 2009

(c) Springer Medizin Verlag 2009

\title{
T. Kälble
}

Klinik für Urologie und Kinderurologie, Klinikum Fulda gAG, Fulda

\section{Alternativ - experimentell - klassisch? Stellenwert verschiedener Therapieverfahren
beim Prostatakarzinom}

Für die Therapie des Prostatakarzinoms existieren mit „watchful waiting“, „active surveillance“, der radikalen Prostatektomie, der Radiotherapie und der Hormontherapie evidenzbasierte Therapieverfahren mit Empfehlungsgraden von meist $\mathrm{A}$ oder B [1]. Dennoch werden v. a. bei der primären lokalen Therapie des Prostatakarzinoms auch andere Behandlungsformen eingesetzt wie hoch fokussierter Ultraschall (Hifu), Kryotherapie oder interstitielle Radiofrequenzablation, die in den Guidelines der EAU 2009 noch als experimentell bezeichnet werden [2]. Darüber hinaus kommen Therapieverfahren mit minimalem Evidenzgrad zum Einsatz wie beispielsweise die Galvanotherapie in mehreren Sitzungen, die von den Patienten in der Regel selbst bezahlt werden müssen.

Im folgenden Heft von „Der Urologe“ werden verschiedene alternative bzw. experimentelle lokale Therapieverfahren des Prostatakarzinoms unter Berücksichtigung der aktuellen Datenlage bzw. eigener Erfahrung dargestellt, mit dem Ziel, uns Urologen Grundlagen für eine umfassende Aufklärung unserer Patienten an die Hand zu geben, die nach Studium des Internets nicht selten eine differenzialtherapeutische Bewertung auch experimenteller Therapieverfahren verlangen. Das Heft wird ergänzt durch eine Übersicht über den aktuellen Stand verschiedenster Formen der immunmodu- latorischen Therapie, die meist beim hormonrefraktären Prostatakarzinom zum Einsatz kommen.

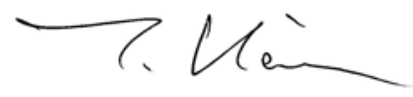

T. Kälble

\section{Korrespondenzadresse}

Prof. Dr. T. Kälble

Klinik für Urologie und Kinderurologie, Klinikum Fulda gAG

Pacelliallee 4, 36043 Fulda

t.kaelble@klinikum-fulda.de

\section{Literatur}

1. Oxford Centre for Evidence-based Medicine Levels of Evidence (2001) Produced by Bob Phillips, Chris Ball, Dave Sacket, Doug Badenoch, Sharon Straus, Brian Haynes, Martin Dawes (since November 1998)

2. Heidenreich A, Bolla M, Joniau S et al. (2009) EAU Guidelines on Prostate Cancer - European Association of Urology. Eur Urol 53: 68-80 